\title{
Twelve Month Follow-Up Audit of Nigerian Hypertensives on Back Titration
}

\section{Basil N Okeahialam*}

Department of Medicine, Cardiology Sub-Unit 1, Jos University Teaching Hospital, Jos, Plateau State, Nigeria

*Corresponding author: Basil. N. Okeahialam FWACP, Department of Medicine, Cardiology Sub-Unit 1, Jos University Teaching Hospital, Jos, Plateau State, Nigeria, Tel: 99907 25799; E-mail: basokeam@yahoo.com; okeahbas@unijos.edu.ng

Received date: Dec 30, 2014; Accepted date: Jan 26, 2015; Published date: Jan 31, 2015

Copyright: (c) 2015 Basil N Okeahialam. This is an open-access article distributed under the terms of the Creative Commons Attribution License, which permits unrestricted use, distribution, and reproduction in any medium, provided the original author and source are credited.

\begin{abstract}
Introduction: An audit of a hypertension service in Jos, Nigeria revealed the possibility of back titration of antihypertensive therapy without untoward effect. The impact was reduction of disease economic burden. Consequently a deliberate policy of back- titration was adopted in those who have remained well controlled for $>12$ months.
\end{abstract}

Objective: To see how low dosages could go without compromising control.

Methods: All consenting hypertensive clients in this specialist hypertension clinic who had been controlled for 12 months or longer on regular follow-up(between July and September 2011); with no heart failure, renal failure, stroke or myocardial infarction ( $<6$ months) were enrolled. Drugs were sequentially back-titrated starting with 1 drug in those on multiple drugs. Follow up continued in the usual manner, and if controlled by the next visit a further dose lowering was advised until loss of control when dose was promptly returned to the lowest maintaining control.

Results: There were 41 patients initially. 2 did not follow up after the first back-titration visit leaving 39 as the subject of this analysis; 14 of whom were males. Their ages ranged from 40 to 91 years and they had been consistently controlled (BP $<140 / 90 \mathrm{mmHg}$ ) for between 12 to 95 months. 18 remained normal at various stages of back-titration; in 5 (3F, 2M) all drugs had been completely withdrawn. In 21, control was lost in the course of backtitration and promptly recovered by returning to previous dose that controlled BP. Those who were successfully back-titrated to no drug were mostly on one drug at low doses.

Conclusion: After a minimum of 12 months of sustained BP control, it is possible to back-titrate drug dosages in about $50 \%$ of this hypertensive cohort, a quarter of whom went completely without drugs for 1 year. The exact mechanism is unknown, but the benefit is in the psychological relief of lower pill burden and reduced cost of treatment. This observation should be extended to other cohorts to prove its applicability; advisedly only under expert care.

Keywords: Hypertension, Treatment, Back titration, Dose, Nigeria

\section{Introduction}

The question of whether pharmacotherapy for systemic arterial hypertension should be life-long has continued to agitate the mind of care givers and patients alike. Whereas it is possible in some cases to reduce dose or discontinue drug(s) outright, relapse of high blood pressure is known to occur after some time [1]. All the same, the attitude of step down or outright discontinuation of anti-hypertensive pharmacotherapy appears to be safe provided close monitoring continued [2]. In an audit of hypertension care in a specialized care facility, the author observed that some patients remained controlled despite self-imposed drug holidays or physician initiated dose reduction following therapy induced hypotensive features [3]. Some earlier workers had called for future studies to shed light on how long and intensively hypertensives could be treated before discontinuation of therapy can be embarked upon [4].

Some authors feel that once good response has occurred with antihypertensive pharmacotherapy and maintained for a reasonable period, medication could be down-titrated [5]. Not certain what period could be judged reasonable and with the call to determine how long and intensively patients could be treated before discontinuation, the author felt the need to make further contribution on the subject. With the experience reported in [3], a deliberate policy of backtitration was embarked upon in the facility. This was with a view to determine how successful physician initiated back-titration without hypotensive features would be in an African hypertensive population. The findings should guide future actions and ameliorate patient psychological and economic burden arising from the disease and its pharmacotherapy.

\section{Methodology}

\section{Study setting and population}

The author operates a private facility providing secondary and tertiary level specialist service to individuals with cardiovascular disease; majority of who is hypertension related. Patients are referred in some cases by primary care physicians but mostly come on their own volition or following information from friends and relations who know the facility for providing such level of service. The patients are made up of a few non-Nigerian Africans, but in the main Nigerians of 
Page 2 of 3

all ethnicities resident in Plateau State; but also from as far as Taraba, Bauchi, Benue, Nassarawa, Kaduna, Niger, Kogi States and the Federal Capital Territory. A few come from the South-Eastern States of Imo, Abia, Anambra, Enugu and Ebonyi at the instance of their relations residing in Jos. They cut across the gender, ethnic, religious and socioeconomic divide. The mode of operation of the facility as it applies to hypertensives has been earlier reported [3]. Briefly on presentation, hypertensives are evaluated and therapy initiated on an individualized basis. Follow up varies in duration depending on control status to give room for dose intensification or modification. Once controlled, patients are seen quarterly on regular follow-up visits.

\section{Study method}

All hypertensives with no heart failure, kidney failure or recent $(<6$ months) stroke or myocardial infarction encumbrances; and had been controlled (Blood Pressure $<140 / 90 \mathrm{mmHg}$ ) continuously for 12 months or longer were included in the study. Those who attended between July 1st and September 30th 2011 were enlisted to avoid being considered more than once and followed up. Drugs were sequentially back-titrated after explanation and consent; starting with one in those on multiple drugs. Those on one drug had dose halved or interval of intake increased. Follow up continued in the usual manner and if controlled based on blood pressure recorded in standard sphygmomanometric fashion by next visit, further dose lowering was advised. This went on until loss of control when dose was promptly returned to the previous dose maintaining control. Age and gender were extracted from their records. All data were anonymised before analysis to ensure confidentiality and conform with laid down standards for human research.

\section{Results}

Forty one patients satisfied the inclusion criteria, and were followed up for 12 months. Two did not follow up after the first back-titration visit leaving 39 as the subject of this analysis. They were 14 males and 25 females. Their ages ranged from 40 to 91 years and they had been consistently controlled for periods ranging from 12 to 95 months.

Eighteen remained normal and were at different stages of back titration. In 5 out of this $18,(2 \mathrm{M}, 3 \mathrm{~F})$ it was possible to withdraw drugs completely in the course of 12 months follow up. In the remaining 21, control was lost in the course of back titration; which promptly normalized by returning to the previous dose that controlled their blood pressures (Table 1). Those who were successfully back-titrated to no drug were mostly on single drug, low dose regimens and had been normal all along with gradual dose reduction.

\begin{tabular}{|c|c|c|c|c|}
\hline S/No & Age (y) & Sex & Drugs at onset & $\begin{array}{l}\text { Drugs at end of } 12 \\
\text { months }\end{array}$ \\
\hline \multicolumn{5}{|c|}{ A - Lost control in the course of back titration } \\
\hline 1 & 73 & M & Mod/Ate 50/Pra1 & Mod/Ate $25^{* * / P r a ~} 1$ \\
\hline 2 & 40 & $\mathrm{~F}$ & Ate $50 / \mathrm{Mod}^{*}$ & Ate $25^{*} / \mathrm{Mod}^{\star * *}$ \\
\hline 3 & 49 & M & Nor $10 /$ Ate 50 & Nor 10 \\
\hline 4 & 72 & $\mathrm{~F}$ & $\begin{array}{l}\text { Pro 120/Lof 10/Ald } \\
\text { 25/Min }\end{array}$ & Lof 2.5/Ald25/Min \\
\hline 5 & 53 & M & CoD 80/Ate 25 & CoD 80/Ate $25^{* * *}$ \\
\hline 6 & 57 & M & CoD 160/Nor 5/Min & CoD 80/Nor 2.5/Min* \\
\hline
\end{tabular}

\begin{tabular}{|c|c|c|c|c|}
\hline 7 & 44 & $F$ & Nor 5/Mod & Nor 2.5 \\
\hline 8 & 63 & $F$ & Ros 25 & $\operatorname{Ros} 25^{\star}$ \\
\hline 9 & 52 & $M$ & Nor 5/Ald 50 & Nor 2.5/Ald 25* \\
\hline 10 & 59 & $M$ & CoD 80 & CoD 40 \\
\hline 11 & 47 & $F$ & Mod/Ros 25 & $\operatorname{Mod}^{*} / \operatorname{Ros} 25$ \\
\hline 12 & 50 & $F$ & Ate 25/Thi & Ate $25^{\star \star \star} /$ Thi \\
\hline 13 & 76 & $F$ & Nor 5/Min /Ald 50 & Nor 2.5/Min /Ald 50 \\
\hline 14 & 71 & $\mathrm{~F}$ & Nor 5/Ros 25/Esi 25 & Nor 5/Ros 25/Esi 25* \\
\hline 15 & 91 & $M$ & Nor 2.5/Bri & Nor 2.5/Bri ${ }^{* *}$ \\
\hline 16 & 51 & $F$ & Mod/Ate 25 & $\operatorname{Mod}^{*} /$ Ate $25^{\star *}$ \\
\hline 17 & 59 & $M$ & Min/Nor 10 & Min/Nor 5 \\
\hline 18 & 43 & $F$ & Bri & $\mathrm{Bri}^{*}$ \\
\hline 19 & 54 & $\mathrm{~F}$ & Ada 20/Ran 5 & Ada 20/Ran 2.5 \\
\hline 20 & 53 & $M$ & Nor 5 & Nor 2.5 \\
\hline 21 & 55 & $\mathrm{~F}$ & Mod *** & $1 / 2 \operatorname{Mod}^{* * *}$ \\
\hline \multicolumn{5}{|c|}{ B - Remained controlled with back titration continuing beyond 12 months } \\
\hline 1 & 51 & $\mathrm{~F}$ & Mod & Mod *** \\
\hline 2 & 62 & $\mathrm{~F}$ & Ros $50 /$ Nor 5 & $\operatorname{Ros} 25^{*}$ \\
\hline 3 & 50 & $\mathrm{~F}$ & Nif 20/Ald 50 & Nif 10 \\
\hline 4 & 59 & $\mathrm{~F}$ & Nor 5 & Nor $2.5^{\star *}$ \\
\hline 5 & 54 & $\mathrm{~F}$ & CoD 80/Nor 5 & CoD 40 \\
\hline 6 & 47 & $\mathrm{~F}$ & Ran 5/Mod & Ran $2.5^{\star *} /$ Mod \\
\hline 7 & 64 & M & Nif 40/Env 10/Esi 50 & Nif 20/Env 10/Esi 25 \\
\hline 8 & 69 & $\mathrm{~F}$ & Mod/Aldo 500 & Mod*/Aldo 125 \\
\hline 9 & 63 & $F$ & Mod/Ate 25/Ald 25 & Mod*/Ate 25 \\
\hline 10 & 58 & $M$ & Ate $25 / \mathrm{Ple} 5$ & Ate $25^{\star * *} /$ Ple 5 \\
\hline 11 & 56 & $\mathrm{~F}$ & Ald 25* & Ald $^{\star *}$ \\
\hline 12 & 48 & $M$ & Nor $2.5^{\star}$ & Nor2. $5^{\star * *}$ \\
\hline 13 & 46 & $M$ & Bri/Ald 75 & Bri \\
\hline \multicolumn{5}{|c|}{$\begin{array}{l}\text { C - Remained controlled even at back titration to complete drug } \\
\text { withdrawal }\end{array}$} \\
\hline 1 & 55 & $F$ & Ald 25 & No drug \\
\hline 2 & 51 & $F$ & Nor 2.5 & No drug \\
\hline 3 & 71 & M & Ros $25 / \operatorname{Mod}^{\star *}$ & No drug \\
\hline 4 & 54 & $M$ & $\mathrm{Bri}^{* *}$ & No drug \\
\hline 5 & 40 & $F$ & Mod & No drug \\
\hline
\end{tabular}

Table 1: Drugs at onset of back titration and status at the end of 12 months 


\begin{abstract}
Abbreviations: Mod: Moduretic; Ate: Atenolol; Pra: Prazosin; Nor: Norvasc; Pro: Propranolol; Lof: Lofral; Ald: Aldactone; Min: Miniplus; CoD: Co-Diovan; Ros: Rosart; Nif: Nifedipine; Thi: Thiapril; Esi: Esidrex; Bri: Brinerdin; Ran: Ranopril; Env: Envas; Aldo: Aldomet; Ple: Plendil; Ada: Adalat
\end{abstract}

Numbers beside drug names indicate total daily dose in mg. Note that Moduretic, Brinerdin, Miniplus and Thiapril have no specific doses beside them as they are fixed standard formulations.

${ }^{\star}$ Drug given every other day, ${ }^{\star *}$ Drug given every third day, ${ }^{* *}$ Drug given weekly.

\section{Discussion}

This work could be considered preliminary; but it has shown that after 12 months of consistent blood pressure control, it is possible to reduce drug doses to varying degrees. This may provide the answer to the poser of Schmieder and Rockstroh [4] on how long on control before withdrawal can be attempted. In a reasonable number, it is possible (especially in those on single low dose regimens) to completely withdraw treatment; and have them remain normal for another 12 months. This will translate into psychological relief of lower pill burden and reduced cost of treatment. How far this can go on would expectedly vary as hypertension is known to be polygenic and multifactorial in pathogenesis.

In 2001, Salako et al. [6] reported decreased dose requirement of anti-hypertensives with age. Finding that hypertensives required lower doses to remain controlled as they aged, they concluded that with a reduced metabolism drugs accumulate in the elderly resulting in marked blood pressure fall. The implication is that lower doses would still accumulate to levels that can still keep blood pressures normal.

In 1999, Gonzalez-Juarnatey [7] reported successful step down of Enalapril therapy in hypertension. In the experience here all drug classes were involved (Table 1). This is corroborated by the experience of Finnerty where reducing the number of drugs in those whose control was maintained by various combinations did not necessarily result in loss of control [8]. Apart from the age related metabolic changes proffered by Salako et al [6], some earlier workers had considered re-setting of the barostat mechanism [9]. It does appear that just like the disease hypertension itself is multifactorial, the mechanisms permitting dose reduction after a reasonable period of control are various [1]. It is known that peripheral arteries that maintain peripheral resistance are muscular, with the smooth muscle layer being significant [10]. Arterial hypertension goes with remodelling of arteries [11] which results in wall stiffness. The result is increase in pulse wave velocity. Successful treatment of hypertension results in arterial stiffness reduction, an experience with most drug classes. When blood pressure is controlled for long, arterial stiffness indexed by pulse wave velocity is known to drop [12]. This occurs by specific relaxation of smooth muscles in the vascular wall, vasodilatation and reduction in accumulation of collagen that may have been laid down in the course of the disease [10].

\section{Conclusion}

In conclusion after a minimum of 12 months of sustained blood pressure control, it is possible to back-titrate drugs dosages successfully in about half of this African hypertensive cohort; in a few completely to life style measures only. Operating via multiple mechanisms the benefit is psychological relief of lower pill burden and improved pharmacoeconomics. It is worth considering as a standard of care provided it is embarked upon only under expert care and continued follow-up with relevant life style modifications. This should be more relevant in low to medium income countries where health expenses are largely out of pocket and affordability is a big issue.

This work is however limited by the small sample size occasioned by the size of the health facility and should be replicated in a bigger health facility. Also oscillometric devices that measure aortic pulse wave velocity from the brachial cuff [13] were not available to the author. Therefore aortic pulse wave velocity which provides information on central aortic blood pressure; a better measure of cardiovascular risk then brachial blood pressure, could not be determined. The reduction in drug doses may have been made more manifest. It has been shown in the work of Sharman et al. [14] that guidance of hypertension treatment with central blood pressure resulted in less use of drugs to achieve blood pressure control than conventional brachial cuff measurements.

\section{References}

1. Schmieder RE, Rockstroh JK, Gatzka CD, Rüddel H, Schächinger H (1997) Discontinuation of antihypertensive therapy: prevalence of relapses and predictors of successful withdrawal in a hypertensive community. Cardiology 88: 277-284.

2. Schmieder RE, Rockstroh JK, Messerli FH (1991) Antihypertensive therapy. To stop or not to stop? JAMA 265: 1566-1571.

3. Okeahialam BN (2009) Stepping down anti-hypertensive therapy: An initial Nigerian experience. Nig J Card 6: 11-13.

4. Schmieder RE, Rockstroh JK (1990) When is discontinuation of antihypertensive therapy indicated? Cardiovasc Drugs Ther 4: 1487-1494.

5. Kaplan NM (1994) Treatment of Hypertension: Drug Therapy. In: Kaplan NM (ED). Clinical Hypertension (6thedn.) Williams and Wilkins. Baltimore 191-280.

6. Salako BL, Ajayi SO, Kadiri S, Arije A (2001) Decreased dose requirement of anti-hypertensive agents with age. Trop Card 27: 3-6.

7. Gonzalez-Juanatey JR, Reino AP, Garcia-Acuna JM, Gonzalez-Juanatey C, Valdes L et al. (1999) Step-Down of Enalapril Treatment for Arterial Hypertension 34: 1287-1292.

8. Finnerty FA Jr (1990) Stepped-down therapy versus intermittent therapy in systemic hypertension. Am J Cardiol 66: 1373-1374.

9. Thrum RH, Smith WM (1967) On resetting of "barostats" in hypertensive patients. JAMA 201: 301-304.

10. Cameron JD, Asmar R, Struijker-Bondier H, Shirai K, Sirenko Y, et al. (2013) Current and future initiatives for vascular health management in clinical practice. Vascular Health and Risk Management 9: 255-264.

11. Nilsson PM, Boutouyrie P, Laurent S (2009) Vascular aging: A tale of EVA and ADAM in cardiovascular risk assessment and prevention. Hypertension 54: 3-10.

12. Kotovskaya Y, Kobalava Z, Vialov S, Vialov I (2005) Peripheral arteries. Properties in the elderly patients. Am J Hypertens 18: 16A.

13. Hametner B, Wassertheurer S, Kropf J, Mayer C, Eber B, et al. (2013) Oscillometric estimation of aortic pulse wave velocity: comparison with intra-aortic catheter measurements. Blood Press Monit 18: 173-176.

14. Sharman JE, Marwick TH, Gilroy D, Otahal P, Abhayaratna WP, et al. (2013) Randomized trial of guiding hypertension management using central aortic blood pressure compared with best-practice care: principal findings of the BP GUIDE study. Hypertension 62: 1138-1145. 